\title{
RAMAN SPECTROSCOPY CHARACTERIZATION OF AMORPHOUS CARBON COATINGS FOR COMPUTER HARD DISKS
}

\author{
Final report of ER-LTR CRADA Project
}

LBNL BG 92-177, CRADA signed October 15, 1992

\begin{abstract}
OF FINAL TECHNICAL REPORT
Amorphous carbon films are used as protective coatings on magnetic media to protect the magnetic layer from wear and abrasion caused by the read/write head during hard disk drive start-up and operation. A key requirement in increasing the storage capacity and reliability of hard-disk drives is improving the performance of these coatings. This cooperative agreement used optical characterization techniques developed at LBNL to study thin-film hard disk media produced by Seagate Technology, major US hard drive manufacturer. The chief scientific goal was relating quantitatively the results of the optical characterization to the underlying chemical structure of the overcoat. In a collaboration with Seagate, LBNL, and Cambridge University, optical and electron-based characterization were used to evaluate the chemical structure of overcoats. The $\mathrm{sp}^{3}$ fraction of the sputtered amorphous carbon films was measured quantitatively for the first time and related to the optical spectroscopy results. This work and other selected aspects of the research performed under the agreement were presented at technical meetings and published in the open literature. The chief technical goal was designing manufacturing processes for the protective carbon overcoat for use in new generations of Seagate disk drives. To this end, joint research carried out under this agreement enabled Seagate to speed development of new coatings which are currently being used in the production of disk media in Seagate's disk-media manufacturing plants in Fremont, CA.
\end{abstract}

The project was begun with the signing of the Cooperative Research and Development Agreement (CRADA) on 10/16/92 (beginning of FY93). The project was substantially complete by the end of FY95 (9/30/95). A small project carryover was spent in FY96 and the project was closed out on 9/30/96.

There is no Proprietary or Protected CRADA Information in this report. 


\section{BACKGROUND}

Hard disk drives are an important part data storage technology and are, for example, critical components of personal computers. During disk operation, the $\mathrm{read} /$ write head flies a tiny distance over the disk surface on a cushion of air. When the disk is turned off, the head skips to a stop on the disk surface. Without a protective coating the magnetic layer would wear out from abrasion from the read/write head and the digital information would be lost. Therefore, a very thin, hard, wear-resistant coating is required. Amorphous carbon is relatively cheap and mechanically advantageous and is used in the industry for this coating. However, small changes in the deposition conditions, both intentional and unintentional, influence the structure, and hence the mechanical performance of the coating. Moreover, the coating has to be ever thinner to keep up with the aggressive disk density trend line, which doubles every $1-2$ years. A critical component of making a competitive disk drive product is designing and implementing an overcoat process that makes a mechanically sound protective coating with high yield.

Researchers at Lawrence Berkeley National Laboratory (LBNL) and Seagate Technology, a firm in the San Francisco Bay Area that manufactures computer disk drives, worked together in this project to develop new types of amorphous carbon coatings that can be applied during the production of a hard disk's thin magnetic layer. $\mathrm{C}$ The chemical and physical properties of amorphous carbon vary with processing in ways that are not well understood. Critical to achieving the goal of this collaboration was the ability to characterize the carbon coatings produced at the atomic level. Such characterization elucidates critical relationship between atomic structure and properties in a material and can lead to ways of optimizing manufacturing processes to improve the material's performance. The project used state-of-the-art, non-destructive spectroscopic techniques that were shown to be able to measure important chemical and physical properties of amorphous carbon coatings.

\section{PROJECT GOALS AND TASKS}

\section{Scientific Goal}

The scientific goal of this project was to understand the relationship in amorphous carbon films between the results of various optical spectroscopies (Raman scattering, refractive index measurements, photoluminescence), physical properties and chemical composition (fraction of $\mathrm{sp}^{3}$ bonds, hydrogen content, density), mechanical properties (hardness, elastic modulus), and performance in hard disk drives.

\section{Technical Goal}

The technical goal of this project was to use the scientific understanding mentioned above with respect to structure-property relationships in amorphous carbon to improve the reliability of protective coating applied to hard disk media. The technical challenge involves developing thinner and thinner coatings with good wear performance to the continuous increasing trend of data density in the hard disk market.

\section{Project Tasks}


At the time of the CRADA signing there were seven proposed scientific tasks to be carried out under the agreement.

(1) Study of the photoluminescence (PL) signal that also appears as an interfering background in "tough" films with high hydrogen contents that had been recently developed by Seagate.

(2) Measurements of optical band gap of films produced by Seagate with varying hydrogen content.

(3) Infrared (IR) spectroscopy of films with high $\mathrm{H}$ content.

(4) Study the relationship of the Raman spectra of amorphous carbon films and their mechanical performance in hard disk drives.

(5) Perform annealing studies of films with high $\mathrm{H}$ contents to determine the structural changes.

(6) Attempt to evaluate the $\mathrm{sp}^{2}$ content of the films by performing chemical tests involving fluorination of double bonds using gaseous $\mathrm{XeF}_{2}$.

(7) Measure film stress and adhesion studies

By mutual consent the project team concentrated on tasks (1), (2), and (4). Two additional tasks were added during the agreement period.

(8) Develop optical tools for evaluating media on the production floor.

(9) Perform electron energy loss spectroscopy (EELS) on hydrogenated and nonhydrogenated films to measure the sp3 content quantitatively.

Seagate was obligated to provide LBNL with sputtered disk media made with production and prototype processes and to provide the results of mechanical, optical, and other tests.

\section{PROJECT RESULTS}

\section{Task 1. Photoluminescence in films with high $H$ content}

It became well-established during the project that the addition of addition of hydrogen to the sputtering gas mixture creates protective films for magnetic media with desirable mechanical properties, e.g. low tribochemical and abrasive wear rates. These sputtered films are similar in structure and mechanical performance to the "diamond-like" films made by chemical vapor deposition (CVD), using various hydrocarbons as the carbon source. In this task the effect of hydrogen content on the Raman and photoluminescence spectrum of $\mathrm{Fi}_{\xi}$ hydrogenated amorphous carbon $(\mathrm{a}-\mathrm{C}: \mathrm{H})$
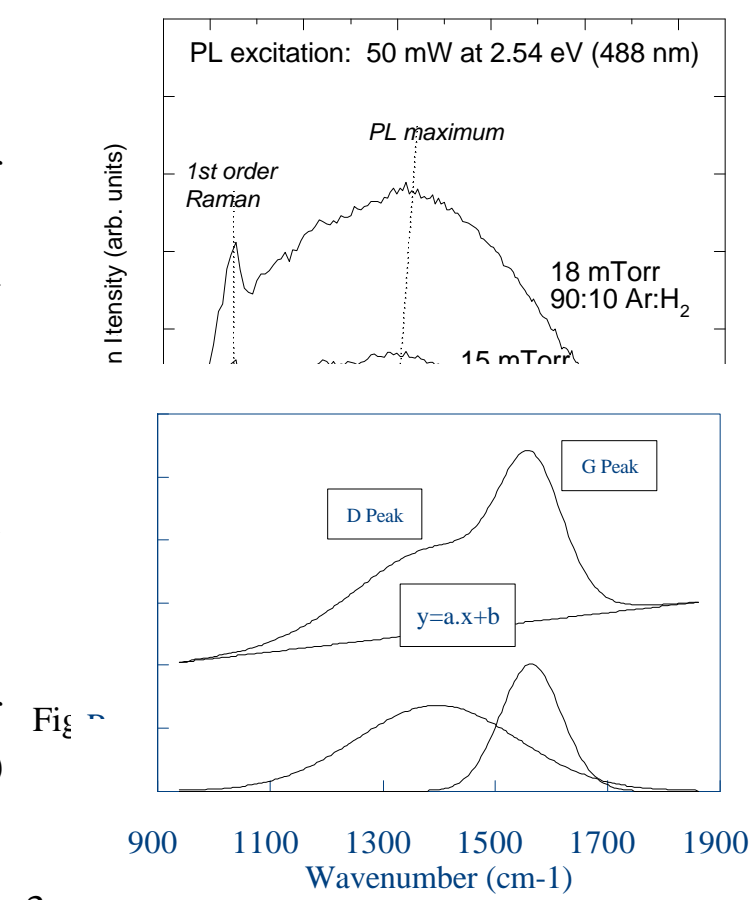
films was studied. This work was reported in detail in B. Marchon et al., "Photoluminescence and Raman spectroscopy in hydrogenated carbon films," IEEE Trans. Magn. 33, 3148-3150 (1997); only a brief summary will be given here.

Figure 1 shows the photoluminescence (PL) spectra obtained at LBNL for carbon films sputtered at various $\mathrm{Ar}: \mathrm{H}_{2}(85: 15)$ pressures. Increasing sputtering pressure leads to an increase in the hydrogen content of the film, and this leads to a strong PL background superimposed on the first order Raman lines. The PL intensity tends to increase with increasing $\mathrm{H}$ concentration due primarily to the saturation of non-radiative recombination sites (e.g., dangling bonds). Figure 2 shows a close-up of the $900-1900 \mathrm{~cm}^{-1}$ region obtained by a Raman spectrometer at Seagate (similar results were obtained at LBNL by similar equipment). A convenient way to estimate the PL intensity is to ratio the slope of the PL background to the fitted Gaussian intensity of the G peak. This slope parameter $S$ has a dimension of length, and is best described in micrometer $(\mu \mathrm{m})$ units. This parameter varies in a systematic way with the hydrogen content as measured by Hydrogen Forward Scattering (HFS), as shown in Figure 3. This quantitative relationship of the Raman/PL spectrum to the hydrogen content (and to the performance of the disk media in disk drives, as discussed below) was a major accomplishment of the project.

\section{Task 2. Band gap and hydrogen content}

While the mechanical properties of amorphous carbon films are believed to be determined by the $\mathrm{sp}^{3}$ interlinks, the electronic structure is believed to be determined by the size of the $\mathrm{sp}^{2}$ domains. These graphitic clusters are suspected to be smaller in films that contain more hydrogen. Using arguments derived from simple Hückel theory, smaller graphitic domains would be expected to have large optical gaps. The hydrogen content in a series of sputtered films was varied as described in Task 1 by increasing the pressure of the 90/10 Ar/ $\mathrm{H}_{2}$ sputtering gas from 10 mTorr to 25 mTorr. The optical constants were measured with a commercial reflection/absorption spectrometer. The results are shown in Fig. 4. The decreasing value of $\mathrm{k}$ in the lower graph with increasing sputtering pressure (and $\mathrm{H}$ content) shows that the $\mathrm{sp}^{2}$ domains are decreasing in size. In other work not discussed in detail here, the results of reflection/absorption spectroscopy were related to process

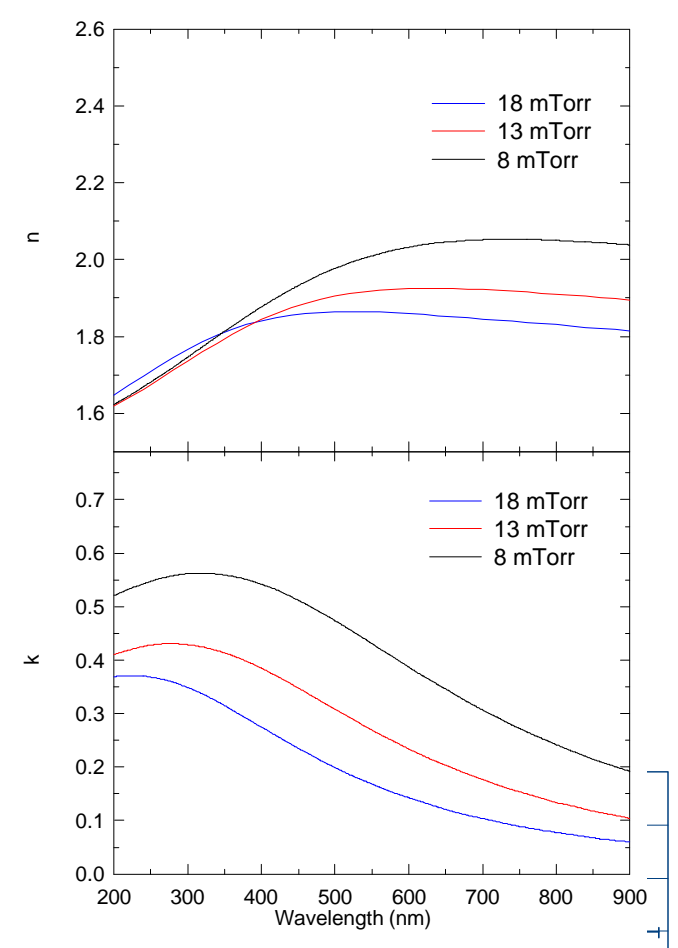

Fig. 4. Beal (n) and imaginary parts of reflective inc

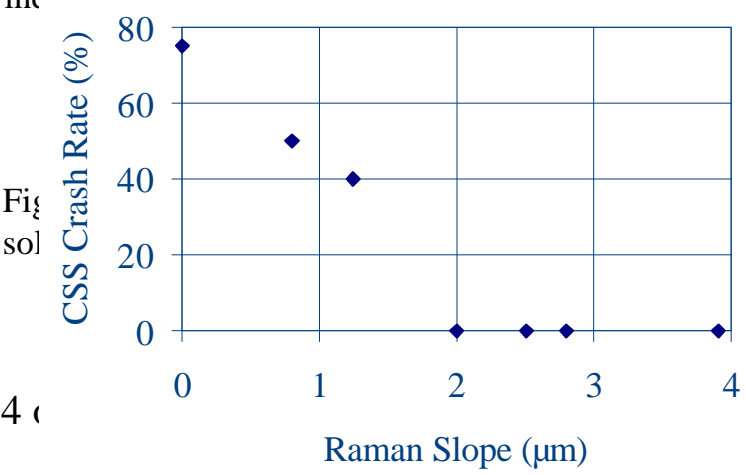


parameters and mechanical tests and used by Seagate to in the evaluation of a-C:H films.

\section{Task 4. Raman/PL spectrum and mechanical performance}

The standard performance test for evaluating hard disk media is contact start-stop (CSS) testing. In a CSS test, a disk drive is turned on and off repeatedly causing the head to take off and land repeatedly on the media. The test is terminated when the disk drive crashes. CSS testing is time-consuming, costly, and destructive. Much of the effort in this project involved predicting the results of CSS testing with non-destructive optical techniques. CSS test results were correlated against Raman spectroscopy parameters (Gand D-band position, width and relative intensity) and optical spectroscopy (band gap). Some of these results are protected CRADA information. Figure 5 shows one unprotected result from B. Marchon et al., IEEE Trans. Magn. 33, 3148-3150 (1997) in which the strength of the PL background (cf. above) is graphed vs. CSS crash rate. Evidently, films with high values of the "Raman slope" (i.e. with relatively high $\mathrm{H}$ contents) lower the CSS crash rate (desirable).

\section{Task 8. Production monitoring equipment}

Based on the relationships between the results of optical spectroscopy and chemical structure (Figs. 1-3) and mechanical performance (Fig. 5), Seagate developed and implemented optical test equipment in their fab. This evaluation method has enabled Seagate to monitor more closely variations in the structure of carbon overcoats in their high-volume production line.

\section{Task 9. Electron Energy Loss Spectroscopy (EELS)}

A collaboration was established with Dr. John Robertson of Cambridge University to evaluate prototype carbon overcoats on Seagate media with electron energy loss spectroscopy (EELS). The EELS technique measures directly the fraction of tetragonal diamond bonds vs. trigonal graphitic bond in the films; the results were used to calibrate optical techniques used at LBNL and Seagate

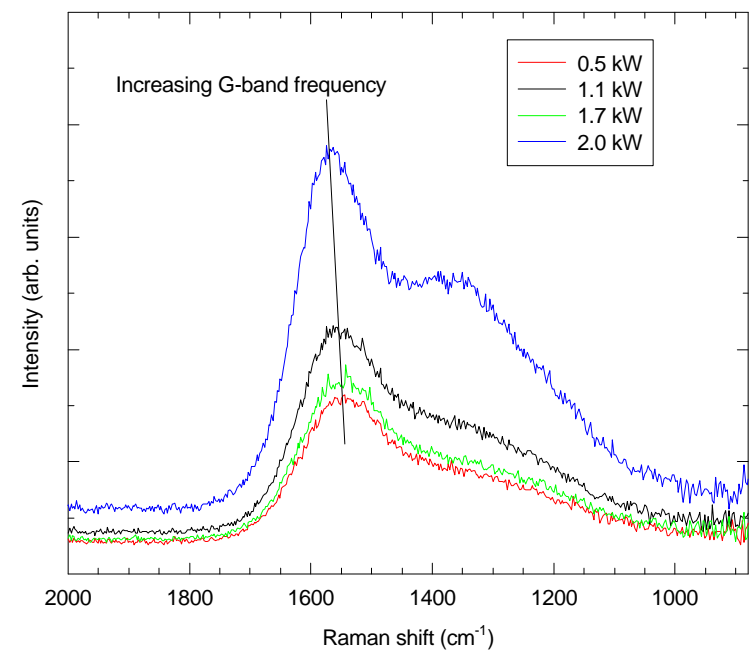

Fig. 6. Raman spectrum of a series of films with decreasing $\mathrm{sp}^{3}$ content and increasing G-band position as the sputtering power is increased (bottom to top).

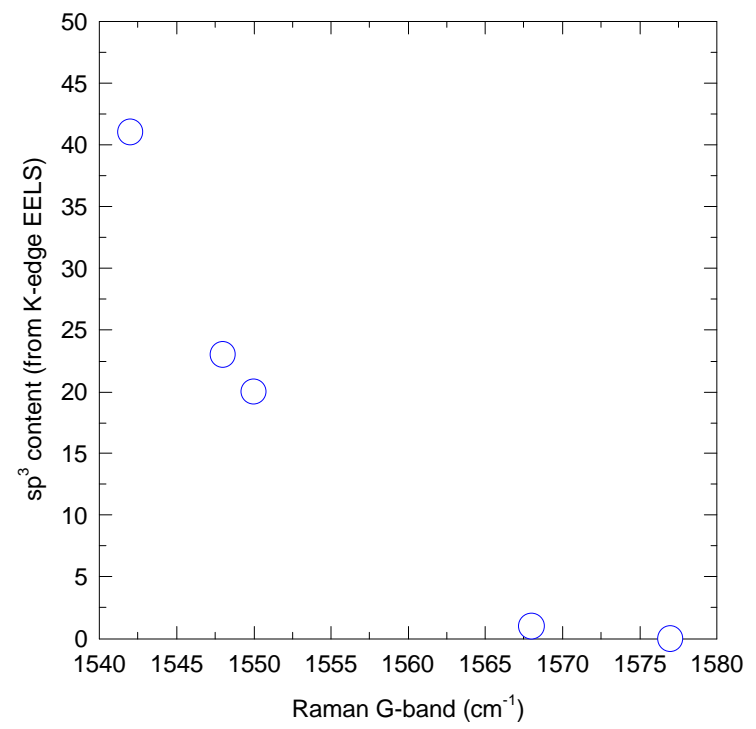

Fig. 7. $\mathrm{sp}^{3}$ content (from EELS) vs. Raman G-band position. 
such as PL and Raman spectroscopy. Some characteristic trends were already clear from Raman studies. For example, as shown in Fig. 6, increasing sample heating was known to both decrease the $\mathrm{sp}^{3}$ content of the films (not desirable for mechanical properties) and increase the frequency of the Raman G-band. What was not known was the quantitative scale of the changes in $\mathrm{sp}^{3}$ content. The EELS measurements were successful and were the first quantitative measure of the $\mathrm{sp}^{3}$ bonding fraction of any Seagate carbon coating (or sputtered a-C:H film, to our knowledge). The relationship between Raman G-band position and $\mathrm{sp}^{3}$ content shown in Fig. 7 was a major accomplishment of this project and was reported in B. Marchon. et al. IEEE Trans. Magn. 33, 3148-3150 (1997).

\section{PROJECT BENEFITS}

\section{Benefits of CRADA to LBNL and DOE}

The basic structure property relationships in carbon films made possible by the joint project (especially the relationship between the EELS and Raman spectra) are helping (1) a multi-laboratory BES effort in developing superhard carbon coatings for application in micromachines (BES Center for Excellence in Synthesis and Processing Project) and (2) were applied to developing a different type of amorphous carbon film for flat panel display applications (ER-LTR CRADA with SI Diamond).

\section{Benefits of CRADA to Seagate}

Seagate developed with some assistance from this project production monitoring equipment based on optical spectroscopy that they are using in the production of hard disk media at their Fremont site. The calibration of optical techniques with EELS has yielded quantitative $\mathrm{sp}^{3}$ fractions for Seagate's amorphous carbon films. This information is being used in the design of better-performing hard disk overcoats.

\section{PROJECT PUBLICATIONS}

J.W. Ager III, "Optical characterization of sputtered carbon films," IEEE Trans. Magn. 29, 259-263 (1993). J. W. Ager III is from LBNL.

J. W. Ager III, B. Marchon, I. Bloomer, and R. Forouhi, "Optical characterization of carbon overcoats," Data Storage Tech. 4, no. 4, 51-54 (1997). B. Marchon is from Seagate Technology.

B. Marchon, J. Gui, K. Grannen, G. C. Rauch, J. W. Ager III, S. R. P. Silva, and J. Robertson, "Photoluminescence and Raman spectroscopy in hydrogenated carbon films," IEEE Trans. Magn. 33, 3148-3150 (1997). J. Gui, K. Grannen, and G. C. Rauch are from Seagate Technology. S. R. P. Silva, and J. Robertson are from Cambridge University.

\section{ACKNOWLEDGMENT}

This research was supported by the Laboratory Technology Research Division (formerly called ER LTT), Office of Energy Research, US Department of Energy under a CRADA (Cooperative Research and Development Agreement) between Lawrence 
Berkeley National Laboratory and Seagate Technology, Fremont, CA under US DOE Contract DE-AC03-76SF00098. 